\title{
Les débuts de la Congrégation des Soeurs Grises de Saint-Hyacinthe, 1840-1865
}

\section{Reine Ponton}

Volume 47, 1980

URI : https://id.erudit.org/iderudit/1007107ar

DOI : https://doi.org/10.7202/1007107ar

Aller au sommaire du numéro

Éditeur(s)

Les Éditions Historia Ecclesiæ Catholicæ Canadensis Inc.

ISSN

0318-6172 (imprimé)

1927-7067 (numérique)

Découvrir la revue

Citer cet article

Ponton, R. (1980). Les débuts de la Congrégation des Soeurs Grises de

Saint-Hyacinthe, 1840-1865. Sessions d'étude - Société canadienne d'histoire de

l'Église catholique, 47, 55-61. https://doi.org/10.7202/1007107ar

Tous droits réservés ㄷ Les Éditions Historia Ecclesiæ Catholicæ Canadensis Inc., 1980
Ce document est protégé par la loi sur le droit d'auteur. L'utilisation des services d'Érudit (y compris la reproduction) est assujettie à sa politique d'utilisation que vous pouvez consulter en ligne.

https://apropos.erudit.org/fr/usagers/politique-dutilisation/ 
s.c.H.E.C., Sessions d'études, 47 (1980, pp. 55-61)

\section{Les débuts de la Congrégation des Sceurs Grises de Saint-Hyacinthe, 1840-1865 *}

Pour bien situer dans le temps les débuts de la Communauté des Sœurs Grises de Saint-Hyacinthe, il faut remonter à 1737, alors qu'à Montréal, Marguerite d'Youville, devenue veuve à 28 ans, après avoir payé les dettes léguées par son frivole mari, ayant assurć l'éducation de ses deux fils, futurs prêtres, et se sentant appelée par Dieu à une vocation de charité envers les pauvres, accepta la direction de l'Hôpital Général de Montréal que les Frères Charon ne pouvaient plus assumer. Ainsi se réalisait la prophétie de M. Louis Normant, p.s.s., son confesseur : «Courage, ma fille, vous relèverez une maison sur son déclin ».

Quand Mère d'Youville fut proclamée Bienheureuse, le 3 mai 1959, le Pape Jean XXIII la désignait comme la « Mère à la charité universelle ». Elle avait un cœur ouvert à toutes les misères humaines, et s'ingéniait à trouver moyen, par les industries les plus variées, à porter secours à toutes les indigences. Sa maison accueillit non seulement les pauvres, les malades, les vieillards, mais aussi les enfants abandonnés et les jeunes filles à protéger. C'est de Louis XV que sa congrégation naissante obtint, par l'entremise des Sulpiciens, les lettres patentes reconnaissant son existence légale.

Marguerite d'Youville a longuement étudié Les saintes voies de la Croix de l'abbé Henri-Marie Boudon, et elle est convaincue que la croix est un signe de bénédiction. Aussi les difficultés, les contradictions, la maladie, le feu, les épreuves, tout contribue à sa sanctification personnelle et fait grandir sa famille religieuse. Le charisme de Mère d'Youville, héritage précieux, les Sœurs Grises y participent et elles désirent que leur dévouement ne fasse défaut à aucune nécessité. "Allez chez les Sœurs Grises, disait-on, elles ne refusent jamais rien ».

* Sur le même sujet, on pourra aussi lire, dans le Rapport 1941-42 du 9e congrès annuel de la SCHEC également tenu à Saint-Hyacinthe, la communication de $\mathbf{M}^{\text {me }}$ Émilie Palardy Dupras, "Mère d'Youville et l'épanouissement de son œuvre à Saint-Hyacinthe », pp. 131-137. 
Comment vont-elles, en 1840, répondre à la demande pressante de Messire Édouard Crevier, grand vicaire du district de SaintHyacinthe, qui s'afflige du triste sort des pauvres de sa paroisse? Sans doute apprécie-t-il grandement le zèle des «Dames de Charité », réunies en association depuis 1828, mais il sait que des mères de familles ne peuvent négliger leurs devoirs domestiques, et il souhaite assurer un service permanent auprès des malades et des pauvres. Il se met à l'œuvre sans tarder. Voici ce que raconte, en $1888, \mathrm{M}^{\mathrm{gr}}$ Alexandre Taché, archevêque de Saint-Boniface : "En recueillant mes souvenirs d'enfant, je me souviens qu'un jour de l'automne de 1838, mes compagnons de collège et moi, nous fûmes surpris de voir que l'on transportait des pierres et autres matériaux tout près de la cour où nous prenions nos récréations. La curiosité ne fut pas lente à s'enquérir: quel édifice allait s'élever là ? et qui viendrait y loger? Nous apprîmes que le digne Curé de Saint-Hyacinthe, désirant ouvrir un asile aux malheureux de sa paroisse, allait leur construire une maison qui s'appellerait l'Hôtel-Dieu ${ }^{1}$ ». À cette entreprise, Messire Crevier consacrait une somme de 60,000 francs, héritage reçu d'une tante récemment décédée.

Un événement marqua le début de son entreprise. «Les matériaux destinés à la construction de son hôpital sont préparés à St-Pie, paroisse à proximité, et transportés à Saint-Hyacinthe en radeaux, sur la rivière Yamaska. Or, un jour, on vient dire à Messire Crevier que les radeaux se brisent et que le bois se disperse au loin, emporté par les flots. Pour toute réponse, Messire Crevier se retire et se met en prière. Que se passa-t-il entre le Ciel et lui ? Nul ne fut initié à ce secret; mais ce qui est parfaitement connu, c'est que ce bois entraîné avec violence dans les rapides s'arrêta tout à coup pour se réunir dans une petite anse non loin de l'endroit où il devait être employé ».

Désormais bien assuré que le Seigneur veut le voir poursuivre son entreprise, Messire Crevier presse les travaux, et dès que son hospice, «modeste construction en bois à deux étages », est achevé, " il vient en offrir la direction aux Sœurs Grises avec la dotation de terrain et des rentes pour subvenir à la pension de douze pauvres ${ }^{2} \gg$. Muni de l'approbation de $\mathbf{M}^{\mathrm{sr}}$ Ignace Bourget, devenu évêque de Montréal à la mort de $\mathbf{M}^{a r}$ Jean-Jacques Lartigue, Messire Crevier parle aux Sœurs Grises de Montréal de ses pauvres, de ses malades,

1 L'Hôpital Général de Montréal, tome II, p. 145.

2 Ibid., p. 146. 
et aussi des privations, du travail et de la souffrance qui seront le lot des fondatrices. Une pareille requête surprend d'autant plus qu'en 1840 , les hospitalières n'exerçaient leur dévouement qu'envers les pauvres et les malades vivant à l'Hôpital ; elles ne sortaient que pour de pressantes nécessités et encore, «en voitures fermées ». Les troubles de '37 étaient dans toutes les mémoires et la prudence s'imposait.

En ce temps-là, ouvrir une maison à Saint-Hyacinthe était une véritable aventure, un peu comme, de nos jours, accepter une fondation en Haïti, au Brésil ou en Afrique. La supérieure, Mère Beaubien, parla du projet à ses sœurs - elles étaient alors une trentaine à Montréal - assurant que quatre sœurs iraient à Saint-Hyacinthe, mais on n'y enverrait que des volontaires. La première à s'offrir pour cette mission fut sœur Michel-Archange Thuot, jadis maîtresse des novices, devenue assistante de la supérieure, à qui s'adjoignirent trois jeunes sœurs formées par elle à la vie religieuse : sœurs Honorine Pinsonnault, Tharsile Guyon et Émilie Jauron. Les préparatifs de cette fondation, une première, se firent avec diligence et enthousiasme ; alors comme aujourd'hui «aller en mission » vous auréolait prématurément d'une espèce de nimbe lumineux !

Le jour vint, 6 mai 1840, où les fondatrices quittèrent l'Hôpital Général de Montréal pour venir à Saint-Hyacinthe. Ce ne fut pas un "au revoir » mais un adieu qu'elles dirent à leur Supérieure et à leurs sœurs, puisque la maison de Saint-Hyacinthe ne devait pas être dépendante de celle de Montréal, mais autonome, comme la plupart des monastères du temps. Cette décision, longuement pesée et mûrie, devait marquer aussi les fondations subséquentes : celle de la Rivière Rouge en 1844, celle d'Ottawa en 1845 et celle de Québec en 1849 .

Voici en quels termes le Père André Guay, O.M.I., raconte l'arrivée à Saint-Hyacinthe :

... on arrive enfin à Saint-Hyacinthe. Que vient faire ici cet " enfin ", surprenant, inattendu? C'est qu'en 1840, Saint-Hyacinthe était bien loin, si loin qu'il fallait s'y rendre par bateau. Par bateau! Regardez une vieille carte de la région; entre Montréal et la "Terre promise" aux quatre "missionnaires de la tendresse ", aucune route, pas même un sentier. Rien, rien du tout. Voilà ! Restait le beau et toujours serviable SaintLaurent. C'est pourquoi l'on s'embarque pour Saint-Hyacinthe. Le "Queen " est là, qui attend les voyageurs au quai Victoria, prêt à transporter missionnaires et bagages, mais jusqu'à Ber- 
thier seulement, première étape. À Berthier, le "Saint-Louis » prend la relève et, via le Richelieu, lon remonte jusqu'à Saint-Charles, deuxième étape ! Assez de bateau ! Maintenant, en voiture! Douze milles jusqu'à Saint-Hyacinthe par des routes impraticables. Que c'est donc loin Saint-Hyacinthe ! Il faut vraiment vouloir y aller.

Si on $y$ arrive fourbu, du moins est-on accueilli avec enthousiasme, comme doivent être accueillies de vraies missionnaires de la tendresse précieux cadeau du ciel à la ville naissante. Messire Crevier sait bien faire les choses. À la rencontre des arrivants à Saint-Charles, il a pris soin d'envoyer sept voitures. Vous lisez bien : sept voitures pour quatre personnes. Eh oui ! les Fondatrices ne sont pas parties "aléges " comme l'on disait. Trois futures postulantes se sont jointes à elles - ça promet ! - et deux filles de service. Enfin, un domestique, promotion masculine évidemment... Puis du bagage, don de l'Hôpital Général et des bienfaiteurs de Montréal. Bref, des missionnaires bien équipées.

À Saint-Hyacinthe, on a eu soin de poster des guetteurs pour scruter l'horizon. Tout à coup, un cri retentit : "Les voilà, les Sœurs Grises, les voilà ! "Saint-Hyacinthe y va immédiatement de ses quatre cloches à toute volée. Les élèves du couvent forment une haie le long de la route, les collégiens se groupent dans la cour du collège, voisin de l'Hôtel-Dieu. Et du haut de la tour du même collège, la fanfare exécute ses plus beaux morceaux. Puis, bien sûr, Messire Crevier est là, entouré des notables de la ville. "Nous lavons enfin, notre Hôtel-Dieu au complet! "C'est vrai. Mais il manque les malades. Demain matin ...

Demain matin! Quand même on est missionnaire de la tendresse, après un tel voyage on est brisć. Pcu importe. On n'est pas venu à Saint-Hyacinthe pour se reposer, " on est des Sœurs Grises ". Messire Crevier s'amène donc sans tarder, mandement de Monseigneur Bourget à la main, document qui établit juridiquement et spirituellement dans l'Église une nouvelle communauté. Et puisqu'il faut agir méthodiquement, il s'agit de trouver une supérieure. On en aura grand besoin. Nomination par l'autorité ? Non pas, élections. Sans cabale aucune, Sœur Thuot devient Mère Thuot, élue première supérieure de l'Hôtel-Dieu; puis Sœur Guyon assistante, Sœur Pinsonnault maîtresse des novices - elles viennent vite, vite Sœur Jauron : aucun titre ... mais beaucoup d'ouvrage. "Elle aura soin des pauvres, des malades et de bien d'autres choses", annonce Messire le Curé. On verra à ce qu'elle ne manque 
pas d'ouvrage, en tout cas. Le bon Curé ne se doute pas de ce qui attend pauvre Sœur Jauron, la benjamine des missionnaires ${ }^{3}$.

Après avoir travaillé avec un courage puisé dans la foi et un ardent amour du Seigneur, Mère Thuot fut atteinte par la paralysie en 1845 et mourait en 1850, dix ans seulement après la fondation. Mère Pinsonnault lui succéda à la tête de la jeune congrégation. Elle sut lui préparer des recrues de choix pour l'avenir, et profita du progrès amené par l'érection du chemin de fer entre Saint-Hyacinthe et Longueuil pour renouer avec la maison de Montréal des relations plus fréquentes, fort utiles au petit Institut qui a besoin de lumière et d'appui. On songe sérieusement, à cette époque, à réunir en une même famille religieuse les Sœurs Grises dispersées par les récentes fondations. Mais la tentative d'union se révèle irréalisable. Devant l'échec de ce projet, la Supérieure de Montréal offre à celles des « anciennes» qui le désirent de retourner à leur maison d'origine.

À Saint-Hyacinthe, Sœur Guyon d'abord, puis Mère Pinsonnault répondent à cette invitation. Mère Jauron, la plus jeune des fondatrices, décide de rester, acceptant à l'avance toutes les épreuves qui ne manqueront pas de frapper une œuvre d'à peine quinze ans. C'était en 1854. La nouvelle Supérieure doit assumer les travaux de reconstruction de l'Hôtel-Dieu qui tombe en ruines et est reconnu nettement trop exigu pour les 15 religieuses professes et pour les pauvres qui réclament soin et assistance.

$\mathrm{Au}$ milieu de toute cette activité, Mère Jauron aura la joie de présider à la fondation de deux maisons dépendantes de l'Hôtcl-Dicu : celle de Sorel en 1862 et l'Ouvroir Ste-Geneviève, à Saint-Hyacinthe même en 1864. Sur la demande de Messire Crevier, devenu curé de Marieville en 1852, Mère Jauron négocie et prépare la fondation de l'Hospice Ste-Croix. Cette maison s'ouvre en 1865, année du jubilé d'argent de la congrégation. Au moment de la bénédiction d'ouverture, Mère Jauron n'exerce plus l'autorité ; elle a déjà commencé un apostolat de la souffrance qui durera vingt ans. Nous la considérons comme notre seconde fondatrice: sa courageuse résolution de demeurer à Saint-Hyacinthe assura la survie de la fondation en territoire mascoutain.

À l'imitation de Marguerite d'Youville, Mère Thuot et Mère Jauron ont donné à la communauté une si forte impulsion que les

3 P. André Guay, Missionnaires de la tendresse, Saint-Hyacinthe, L'Accueil, 1980, pp. 23-26. 
Sœurs Grises de Saint-Hyacinthe, après 140 ans d'existence, continuent de servir, avec des moyens limités sans doute - on connaît la situation qui règne dans le domaine hospitalier et celui de l'éducation - mais la flamme de la charité brille toujours dans leur ciel. Je ne vous en citerai qu'une preuve.

La semaine dernière, j'étals à Berlin, New Hampshire, ou l'administration de l'ancien Hôpital Saint-Louis - maintenant Androscoggin Valley Hospital depuis 1971 - célébrait le $75^{\mathrm{e}}$ anniversaire de cette institution, donc, de l'arrivée des Sœurs Grises à Berlin. Toutes les sœurs qui ont œuvré dans cette maison étaient invitées à une fête du souvenir. L'Amicale de l'École des Infirmières avait préparé une rencontre vraiment chaleureuse. J'ai entendu fuser de toutes parts les exclamations joyeuses des anciennes qui se retrouvaient après bien des années et d'autres qui cherchaient sœur une telle, sœur une telle. Il y eut des discours, de beaux gestes de reconnaissance, mais le moment le plus émouvant fut sans contredit le rappel d'un événement qui fit la manchette des journaux en 1963.

Vers 7 heures 30, un matin de décembre, une jeune femme de 28 ans était amenée à l'hôpital à la suite d'un accident de la route. La religieuse infirmière, surveillante de nuit, se rendit compte que la mort avait déjà fait son œuvre, mais son expérience de 20 ans en obstétrique lui fit découvrir que la femme était enceinte et sur le point d'accoucher. Le médecin, alerté immédiatement, constata que le bébé était vivant et pratiqua une césarienne. La petite fille, Cécile, vit le jour trente minutes après la mort de sa mère. Il va sans dire qu'un lien étroit l'unit à la religieuse qui lui a sauvé la vie. Étudiante actuellement au Collège Rivier, à Hudson, la jeune fille est venue rendre témoignage à sa «seconde mère » et elle a déclaré que "la vie est merveilleuse !» Que dirc alors quand ce sont des âmes qui renaissent à l'amitié de Dieu, touchées par le dévouement discret d'une religieuse !

$\mathrm{Au}$ cours de cet entretien, j'ai évoqué trop rapidement le souvenir des Dames de Charité. J'y reviens pour célébrer la cordiale fraternité qui règne depuis 140 ans entre les Dames de Charité et les Sœurs Grises. Elles font cause commune au service des pauvres. Le soir du 7 mai 1840, après les fatigues du voyage et les émotions d'un accueil trop enthousiaste, les fondatrices sont heureuses de se trouver seules dans leur Hôtel-Dieu et de s'y installer pour la nuit. Elles devront d'abord refaire leurs forces, car le premier souper pris 
dans leur nouvelle demeure les attend, préparé et servi par les soins de la seigneuresse, $\mathbf{M}^{\text {me }}$ Jean Dessaulles. C'est là le premier anneau d'une longue chaîne de bienfaits, de délicates prévenances. Si le bazar fut, dans la région de Saint-Hyacinthe, un événement annuel où l'on accourait de partout, et cela de 1846 à 1956, ce fut grâce au zèle des Dames de Charité. Elles le préparaient tout au cours de l'année. Aussi étaient-elles justement fières du succès couronnant leur travail et procurant aux pauvres un peu de bien-être. De nos jours encore, les Dames Auxiliaires poursuivent à l'Hôtel-Dieu de Saint-Hyacinthe les généreuses traditions de leurs devancières et s'ingénient à souligner les grandes fêtes en apportant plaisirs et sourires à tous les chers malades de cette institution. C'est que «l'amour ne passera jamais».

On me reprochera sans doute d'avoir à peine nommé Mgr Bourget. Et pourtant, nous lui devons notre mission d'Église, une mission nette et précise. En vrai pasteur des âmes, il considérait comme un devoir d'être «le père des pauvres et des orphelins, l'œil de l'aveugle, le pied du boiteux »; il le dit si bien dans le mandement d'érection de la communauté des Sœurs Grises de Saint-Hyacinthe. Mais un évêque ne pouvant tout accomplir lui-même doit déléguer certaines de ses responsabilités. Pour un ministère de tendresse auprès des membres souffrants du Christ, où trouver mieux que des femmes au cœur libre d'attaches humaines même légitimes, qui se vouent corps et âme au mieux-être de tous ceux qui sont blessés par le deuil, l'âge, la maladie?

C'est là la véritable vocation de toute Sœur Grise : être constamment à l'écoute des autres pour leur porter secours de façon efficace. Tout cela dans la foi en Celui qui dira, lors du jugement dernier : «Ce que vous avez fait au plus petit d'entre les miens, c'est à moi que vous l'avez fait » (Mt. 25,40).

Sœur Reine PonTon, s.c.s.h.,

Maison Généralice, Saint-Hyacinthe. 